\title{
Evaluation of NSP to Estimate Seismic Deformation: SDF SYSTEMS
}

\author{
By Anil K. Chopra' ${ }^{1}$ and Rakesh K. Goel ${ }^{2}$
}

\begin{abstract}
Investigated in this paper is the approximation in the ATC-40 nonlinear static procedure (NSP) that the earthquake-induced deformation of an inelastic single-degree-of-freedom (SDF) system can be estimated by an iterative method requiring analysis of a sequence of equivalent linear systems. Several deficiencies in the ATC-40 Procedure A are demonstrated. This iterative procedure did not converge for some of the systems analyzed. It converged in many cases, but to a deformation much different than dynamic (nonlinear response history or inelastic design spectrum) analysis of the inelastic system. The ATC-40 Procedure B always gives a unique value of deformation, same as that determined by Procedure A if it converged. These approximate procedures underestimate significantly the deformation for a wide range of periods and ductility factors with errors approaching 50\%, implying that the estimated deformation is about half the "exact" value. Surprisingly, the ATC-40 procedures are deficient relative to even the elastic design spectrum in the velocity-sensitive and displacement-sensitive regions of the spectrum. For systems with a period in these regions, the peak deformation of an inelastic system can be estimated from the elastic design spectrum using the well-known equal displacement rule. However, the approximate procedure requires analyses of several equivalent linear systems and still produces worse results.
\end{abstract}

\section{INTRODUCTION}

A major challenge to performance-based seismic design and engineering of buildings is to develop simple, yet effective, methods for designing, analyzing, and checking the design of structures so that they reliably meet the selected performance objectives. Needed are analysis procedures that are capable of predicting the demands - forces and deformations - imposed by earthquakes on structures more realistically than has been done in building codes. In response to this need, simplified, nonlinear analysis procedures have been incorporated in the ATC-40 and FEMA-274 documents (ATC 1996; FEMA 1997) to determine the displacement demand imposed on a building expected to deform inelastically.

The nonlinear static procedure (NSP) in these documents is based on the capacity spectrum method originally developed by Freeman et al. (1975) and Freeman (1978). It consists of the following steps:

1. Develop the relationship between base shear $V_{b}$ and roof (Nth floor) displacement $u_{N}$ [Fig. 1(a)], commonly known as the pushover curve.

2. Convert the pushover curve to a capacity diagram [Fig. 1(b)], where

$$
\Gamma_{1}=\frac{\sum_{j=1}^{N} m_{j} \phi_{j 1}}{\sum_{j=1}^{N} m_{j} \phi_{j 1}^{2}} ; \quad M_{1}^{*}=\frac{\left(\sum_{j=1}^{N} m_{j} \phi_{j 1}\right)^{2}}{\sum_{j=1}^{N} m_{j} \phi_{j 1}^{2}}
$$

and $m_{j}=$ lumped mass at $j$ th floor level; $\phi_{j 1}=j$ th floor element of the fundamental mode $\phi_{1} ; N=$ number of floors; and $M_{1}^{*}=$ effective modal mass for the fundamental vibration mode.

3. Convert the elastic response (or design) spectrum from

\footnotetext{
Johnson Prof., Dept. of Civ. and Envir. Engrg., Univ. of California, Berkeley, Berkeley, CA 94720.

${ }^{2}$ Assoc. Prof., Dept. of Civ. and Envir. Engrg., California Polytechnic State Univ., San Luis Obispo, CA 93407.
}

the standard pseudoacceleration $A$ versus natural period $T_{n}$ format to the $A-D$ format, where $D=$ deformation spectrum ordinate [Fig. 1(c)].

4. Plot the demand diagram and capacity diagram together and determine the displacement demand [Fig. 1(d)]. Involved in this step are dynamic analyses of a sequence of equivalent linear systems with successively updated values of the natural vibration period $T_{e q}$ and equivalent viscous damping $\hat{\zeta}_{e q}$ (to be defined later).

5. Convert the displacement demand determined in Step 4 to global (roof) displacement and individual component deformation and compare them to the limiting values for the specified performance goals.

Approximations are implicit in the various steps of this simplified analysis of an inelastic multi-degree-of-freedom (MDF) system. Implicit in Steps 1 and 2 is a lateral force distribution assumed to be fixed and based only on the fundamental vibration mode of the elastic system; however, extensions to account for higher mode effects have been proposed (Paret et al. 1996). Implicit in Step 4 is the belief that the earthquakeinduced deformation of an inelastic single-degree-of-freedom (SDF) system can be estimated satisfactorily by an iterative method requiring analysis of a sequence of equivalent linear SDF systems, thus avoiding the dynamic analysis of the inelastic SDF system. The objective of this investigation is to evaluate the accuracy of Step 4, a critical step, of the ATC-40 procedure.

\section{EQUIVALENT LINEAR SYSTEMS}

The earthquake response of inelastic systems can be estimated by approximate analytical methods in the which the nonlinear system is replaced by an "equivalent" linear system. Much of the fundamental work on equivalent linear systems was accomplished over two decades ago (Hudson 1965; Jennings 1968; Iwan and Gates 1979). Now there is renewed interest in applications of equivalent linear systems for the design of inelastic structures. For such applications, the secant stiffness method (Jennings 1968) is being used in the capacity spectrum method to check the adequacy of a structural design [e.g., Freeman et al. (1975), Freeman (1978), Deierlein and Hsieh (1990), Reinhorn et al. (1995)] and has been adapted to develop the "nonlinear static procedure" in the ATC-40 report (ATC 1996) and the FEMA-274 report (FEMA 1997).

The equivalent linear system based on the secant stiffness is reviewed next. Consider an inelastic SDF system with bi- 

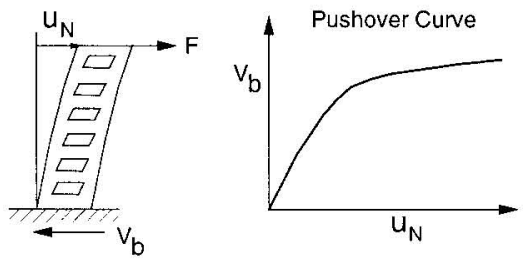

(a)

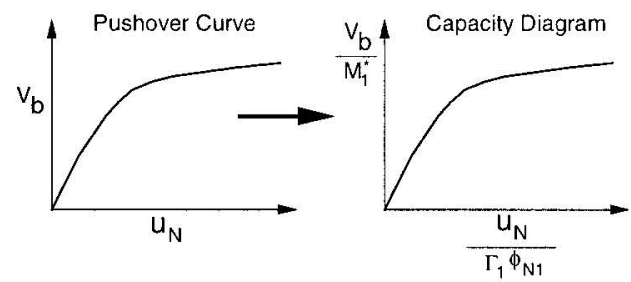

(b)
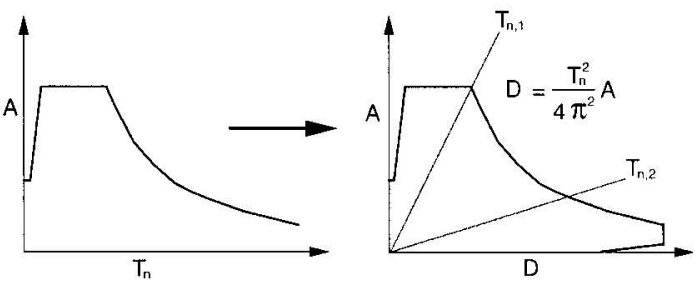

Demand Diagram

(c)

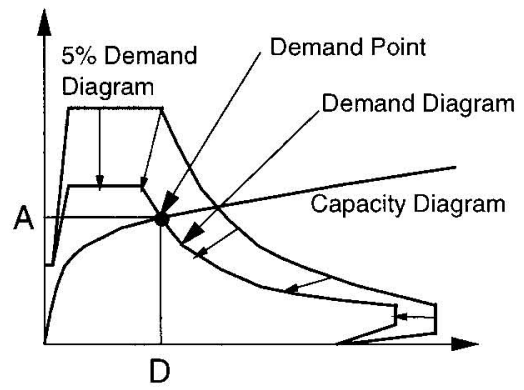

(d)

FIG. 1. Capacity Spectrum Method: (a) Development of Pushover Curve; (b) Conversion of Pushover Curve to Capacity Diagram; (c) Conversion of Elastic Response Spectrum from Standard Format to $A-D$ Format; and (d) Determination of Displacement Demand

linear force-deformation relationship on initial loading [Fig. 2(a)]. The stiffness of the elastic branch is $k$ and that of the yielding branch is $\alpha k$. The yield strength and yield displacement are denoted by $f_{y}$ and $u_{y}$, respectively. If the peak (maximum absolute) deformation of the inelastic system is $u_{m}$, the ductility factor $\mu=u_{m} / u_{y}$. For the bilinear system of Fig. 2(a), the natural vibration period of the equivalent linear system with stiffness equal to the secant stiffness $k_{\text {sec }}$ is

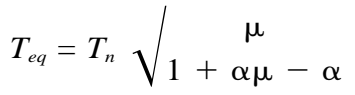

where $T_{n}=$ natural vibration period of the system vibrating within its linearly elastic range $\left(u \leq u_{y}\right)$.

The most common method for defining equivalent viscous damping is to equate the energy dissipated in a vibration cycle of the inelastic system and of the equivalent linear system. Based on this concept, it can be shown that the equivalent viscous damping ratio is (Chopra 1995, Section 3.9)

$$
\zeta_{e q}=\begin{array}{cc}
1 & E_{D} \\
4 \pi & E_{S}
\end{array}
$$

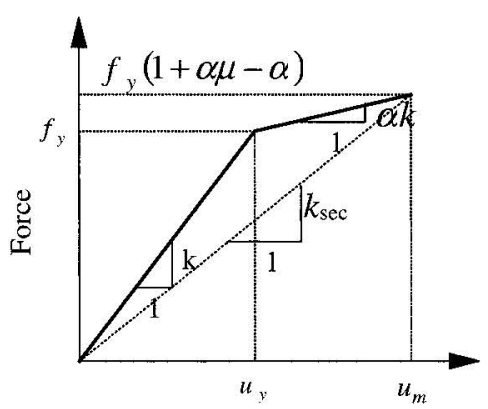

(a)

Deformation

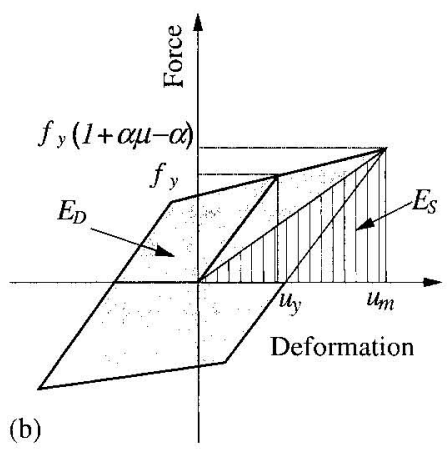

FIG. 2. Inelastic SDF System: (a) Bilinear Force-Deformation Relationship; (b) Equivalent Viscous Damping due to Hysteretic Energy Dissipation

where the energy dissipated in the inelastic system is given by the area $E_{D}$ enclosed by the hysteresis loop [Fig. 2(b)]; and $E_{S}$ $=k_{\mathrm{sec}} u_{m}^{2} / 2$ is the strain energy of the system with stiffness $k_{\mathrm{sec}}$ [Fig. 2(b)]. Substituting for $E_{D}$ and $E_{S}$ in (3) leads to

$$
\zeta_{e q}=\begin{aligned}
& 2(\mu-1)(1-\alpha) \\
& \pi \mu(1+\alpha \mu-\alpha)
\end{aligned}
$$

The total viscous damping of the equivalent linear system is

$$
\hat{\zeta}_{e q}=\zeta+\zeta_{e q}
$$

where $\zeta=$ viscous damping ratio of the bilinear system vibrating within its linearly elastic range $\left(u \leq u_{y}\right)$. For elastoplastic systems, $\alpha=0$ and (2) and (4) reduce to

$$
T_{e q}=T_{n} \vee \mu ; \quad \zeta_{e q}=\begin{aligned}
& 2 \mu-1 \\
& \pi \mu
\end{aligned}
$$

\section{ATC-40 ANALYSIS PROCEDURES}

Contained in the ATC-40 report are approximate analysis procedures to estimate the earthquake-induced deformation of an inelastic system. These procedures are approximate in the sense that they avoid dynamic analysis of the inelastic system. Instead, dynamic analyses of a sequence of equivalent linear systems with successively updated values of $T_{e q}$ and $\hat{\zeta}_{e q}$ provide a basis to estimate the deformation of the inelastic system; $T_{e q}$ is determined by (2) but $\hat{\zeta}_{e q}$ by a modified version of (5)

$$
\hat{\zeta}_{e q}=\zeta+\kappa \zeta_{e q}
$$

with $\zeta_{e q}$ limited to 0.45 . Although the basis for selecting this upper limit on damping is not stated explicitly, ATC-40 states that "The committee who developed these damping coefficients concluded that spectra should not be reduced to this extent at higher values and judgmentally ... set an absolute limit on ... [0.05 $\left.+\zeta_{\text {eq }}\right]$ of about 50 percent.",

The damping modification factor $\kappa$, based primarily on judgment, depends on the hysteretic behavior of the system 


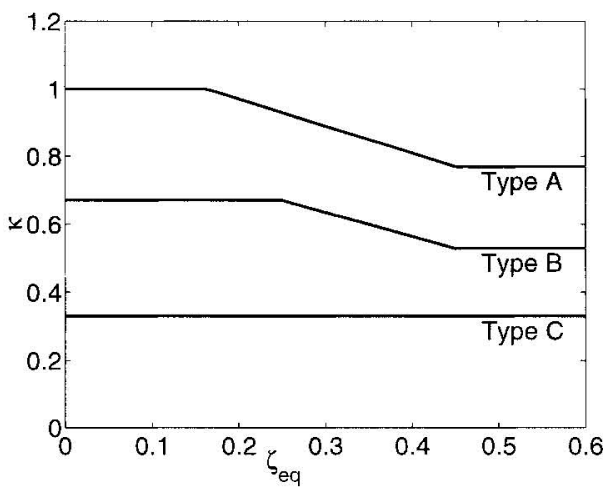

FIG. 3. Variation of Damping Modification Factor with Equivalent Viscous Damping

characterized by one of three types: Type A denotes hysteretic behavior with stable, reasonably full hysteresis loops, whereas Type C represents severely pinched and/or degraded loops; and Type B denotes hysteretic behavior intermediate between Types A and C. ATC-40 contains equations for $\kappa$ as a function of $\zeta_{e q}$ computed by (3) for the three types of hysteretic behavior. These equations, plotted in Fig. 3, were designed to ensure that $\kappa$ does not exceed an upper limit, a requirement in addition to the limit of $45 \%$ on $\zeta_{e q}$. ATC-40 states that "... they represent the consensus opinion of the product development team.' Concerned with bilinear systems, this paper will use the $\kappa$ specified for Type A systems.

ATC-40 specifies three different procedures to estimate the earthquake-induced deformation demand, all based on the same underlying principles but differing in implementation. Procedures A and B are analytical and amenable to computer implementation, whereas procedure $\mathrm{C}$ is graphical and most suited for hand analysis. Designed to be the most direct application of the methodology, Procedure A is suggested to be the best of the three procedures. The capacity diagram is assumed to be bilinear in Procedure B. The description of Procedures A and B that follows is equivalent to that in the ATC40 report except that it is specialized for bilinear systems.

\section{Procedure A}

This procedure in the ATC-40 report is described herein as a sequence of steps:

1. Plot the force-deformation diagram and the 5\%-damped elastic response (or design) diagram, both in the $A-D$ format to obtain the capacity diagram, and 5\%-damped elastic demand diagram, respectively.

2. Estimate the peak deformation demand $D_{i}$ and determine the corresponding pseudoacceleration $A_{i}$ from the capacity diagram. Initially, assume $D_{i}=D\left(T_{n}, \zeta=5 \%\right)$, determined for period $T_{n}$ from the elastic demand diagram.

3. Compute ductility $\mu=D_{i} \div u_{y}$.
4. Compute the equivalent damping ratio $\hat{\zeta}_{e q}$ from (7).

5. Plot the elastic demand diagram for $\hat{\zeta}_{e q}$ determined in Step 4 and read off the displacement $D_{j}$ where this diagram intersects the capacity diagram.

6. Check for convergence. If $\left(D_{j}-D_{i}\right) \div D_{j} \leq$ tolerance $(=0.05)$, then the earthquake-induced deformation demand $D=D_{j}$. Otherwise, set $D_{i}=D_{j}$ (or another estimated value) and repeat Steps 3-6.

\section{Examples}

The ATC-40 Procedure A is implemented to analyze systems with the excitation specified by the elastic design spectrum of Fig. 4, which is the median-plus-one-standard-deviation spectrum constructed by the procedures of Newmark and Hall (1982), as described in Chopra (1995, Section 6.9). This procedure is used to compute the earthquake-induced deformation of the six examples systems listed in Table 1. Considered are two values of $T_{n}(0.5 \mathrm{~s}$ in the acceleration-sensitive spectral region and $1 \mathrm{~s}$ in the velocity-sensitive region) and three levels of yield strength; $\zeta=5 \%$ for all systems. The yield strength for each system is determined from the constant ductility design spectrum for the selected ductility factor (Table 1).

The procedure is implemented for System 5 (Table 1):

1. Implementation of Step 1 gives the 5\%-damped elastic demand diagram and capacity diagram in Fig. 5(a).

2. Assume $D_{i}=D(1.0,5 \%)=44.64 \mathrm{~cm}$.

3. $\mu=44.64 \div 11.16=4$.

4. $\zeta_{e q}=0.637 \times(4.0-1) \div 4.0=0.48$; instead, use the maximum allowable value 0.45 . For $\zeta_{e q}=0.45$ and Type A systems (Fig. 3), $\kappa=0.77$ and $\hat{\zeta}_{e q}=\zeta+\kappa \zeta_{e q}=0.05$ $+0.77 \times 0.45=0.397$.

5. The elastic demand diagram for $39.7 \%$ damping intersects the capacity diagram at $D_{j}=28.18 \mathrm{~cm}$ [Fig. 5(a)].

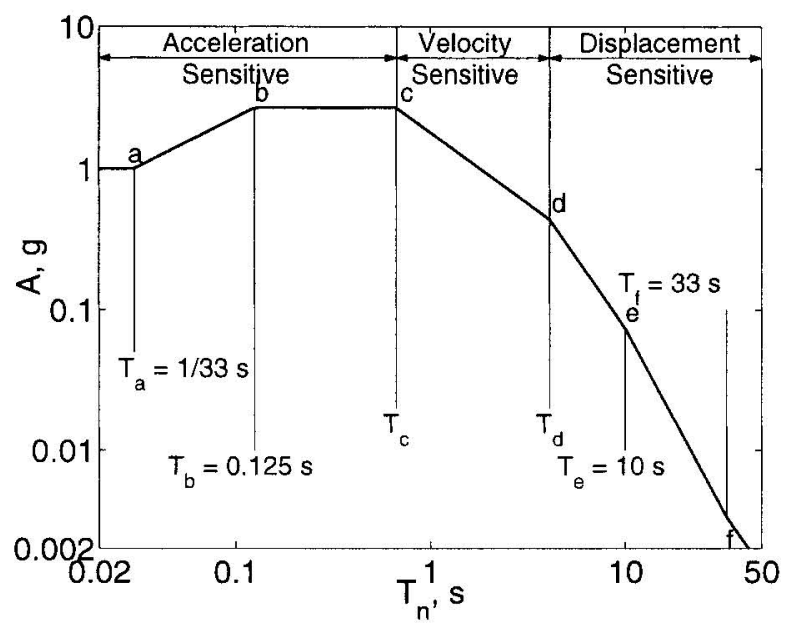

FIG. 4. Newmark-Hall Elastic Design Spectrum

\section{TABLE 1. Properties and Results from ATC-40 Procedure B Analysis of Six Systems for Design Spectrum}

\section{System Properties}

$T_{n}$
$(\mathrm{~s})$

System

1

2

3

4
5

6
(2)

0.5

0.5

0.5

1

1 $f_{y} \div w$

(3)

0.5995

0.8992

1.5624

0.2997

0.4496

0.8992
System Response

$u_{y}$
$(\mathrm{~cm})$
$(4)$
3.7202
5.5803
9.6962
7.4403
11.160
22.321

$\begin{array}{cc}\mu & D_{\text {spectrum }} \\ (5) & (6) \\ 6 & 22.32 \\ 4 & 22.32 \\ 2 & 19.39 \\ 6 & 44.64 \\ 4 & 44.64 \\ 2 & 44.64\end{array}$

Discrepancy

$-53.1$

$-58.6$

$-40.6$

$-5.2$

$-31.7$

$-33.1$ 

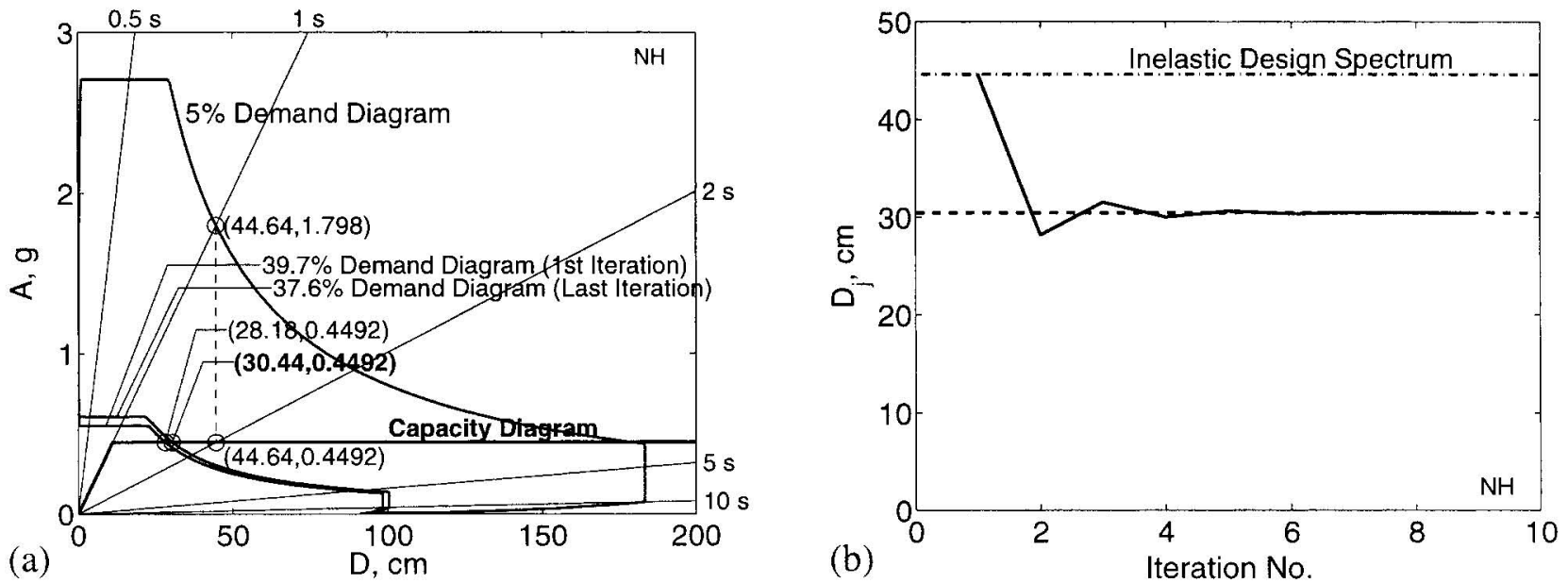

FIG. 5. Application of ATC-40 Procedure A to Example 5 for Elastic Design Spectrum: (a) Iterative Procedure; (b) Convergence Behavior (NH = Newmark-Hall)
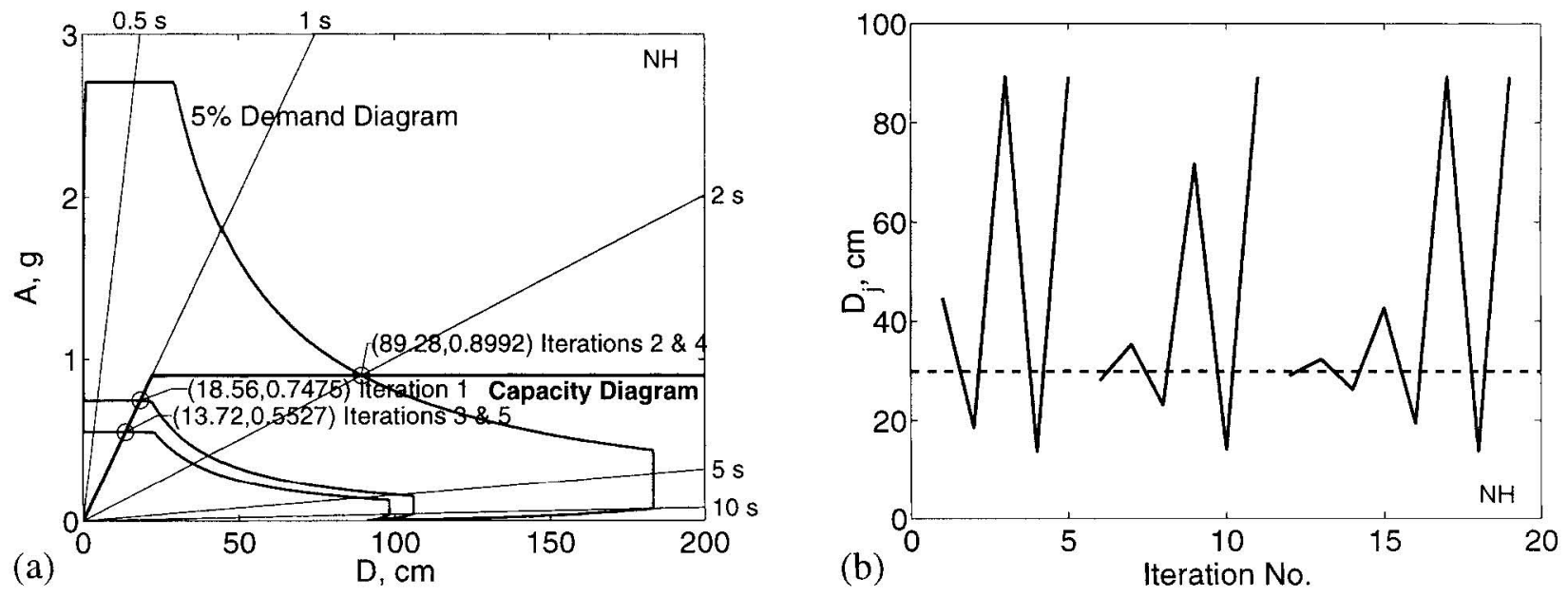

FIG. 6. Application of ATC-40 Procedure A to System 6 for Elastic Design Spectrum: (a) Iterative Procedure; (b) Convergence Behavior (NH = Newmark-Hall)

6. $100 \times\left(D_{j}-D_{i}\right) \div D_{j}=100 \times(28.18-44.64) \div$ $28.18=-58.4 \%>5 \%$ tolerance. Set $D_{i}=28.18 \mathrm{~cm}$ and repeat Steps 3-6.

For the second iteration, $D_{i}=28.18 \mathrm{~cm}, \mu=28.18 \div 11.16$ $=2.52, \zeta_{\text {eq }}=0.637 \times(2.52-1) \div 2.52=0.38, \kappa=0.84$, and $\hat{\zeta}_{e q}=0.37$. The intersection point $D_{j}=31.55 \mathrm{~cm}$, and the difference between $D_{i}$ and $D_{j}$ is $10.7 \%$, which is greater than the $5 \%$ tolerance. Therefore, additional iterations are required; results of these iterations are summarized in Chopra and Goel (1999). The deformation demand at the end of the iteration process is $D_{j}=30.44 \mathrm{~cm}$.

Determined directly from the inelastic design spectrum, constructed by the procedures of Newmark and Hall (1982) as described in Chopra (1995, Section 7.10), the "reference" value of deformation is $D_{\text {spectrum }}=44.64 \mathrm{~cm}$ and the discrepancy $=100 \times(30.44-44.64) \div 44.64=-31.8 \%$.

Fig. 5(b) shows the convergence behavior of the ATC-40 Procedure A for System 5. Observe that the iterative procedure converges to a deformation value much smaller than the reference value. Thus, convergence is deceptive because it can leave the erroneous impression that the calculated deformation is accurate. In contrast, a rational iterative procedure should lead to the "exact" result after a sufficient number of iterations. In this case, the initial estimate $D_{1}=44.64 \mathrm{~cm}$ is the deformation of the corresponding elastic system, which is also equal to the deformation of the inelastic system because $T_{n}$ is in the velocity-sensitive region of the spectrum. However, the ATC-40 procedure fails to recognize this coincidence and proceeds with iterations when it should not and worsens the deformation value in the process.

The procedure is next implemented for System 6 (Table 1). The results are presented in Fig. 6 where it is shown that the procedure fails to converge. In the first iteration, the $33 \%$ damped elastic demand diagram intersects the capacity diagram in its linear-elastic region [Fig. 6(a)]. In subsequent iterations, the intersection point alternates between 13.72 and $89.28 \mathrm{~cm}$ [Fig. 6(b)]. To examine if the procedure would converge with a new starting point, the procedure was restarted with $D_{i}=28 \mathrm{~cm}$ at iteration number 6 . However, the procedure diverges very quickly as shown by iterations 6-11 [Fig. 6(b)], ending in an alternating pattern. Detailed calculations for all systems are available in Chopra and Goel (1999).

\section{Procedure B}

This procedure in the ATC-40 report is described herein as a sequence of steps:

1. Plot the capacity diagram. 
2. Estimate the peak deformation demand $D_{i}$. Initially assume $D_{i}=D\left(T_{n}, \zeta=5 \%\right)$.

3. Compute ductility $\mu=D_{i} \div u_{y}$.

4. Compute equivalent period $T_{e q}$ and damping ratio $\hat{\zeta}_{e q}$ from (2) and (7), respectively.

5. Compute the peak deformation $D\left(T_{e q}, \hat{\zeta}_{e q}\right)$ and peak pseudoacceleration $A\left(T_{e q}, \hat{\zeta}_{e q}\right)$ of an elastic SDF system with vibration properties $T_{e q}$ and $\hat{\zeta}_{e q}$.

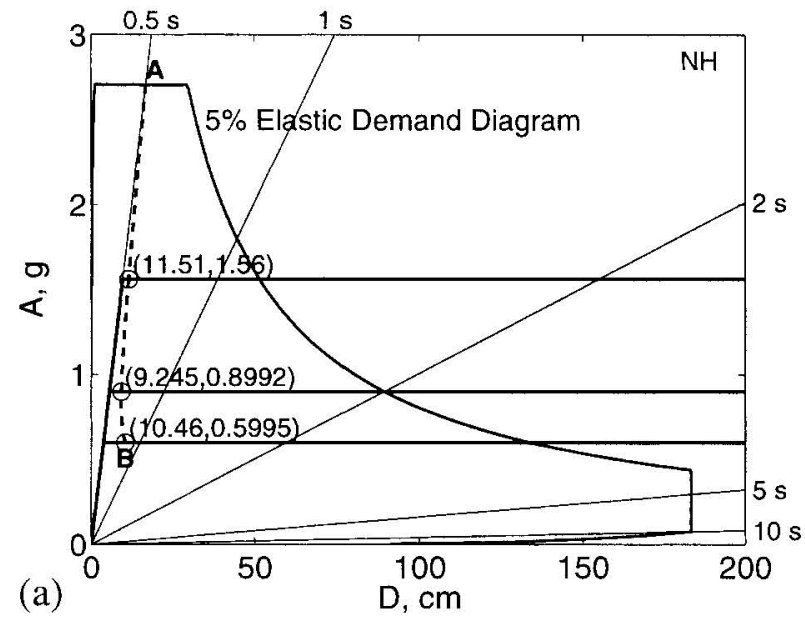

6. Plot the point with coordinates $D\left(T_{e q}, \hat{\zeta}_{e q}\right)$ and $A\left(T_{e q}\right.$, $\left.\hat{\zeta}_{e q}\right)$.

7. Verify that the curve generated by connecting the point plotted in Step 6 to previously determined, similar points intersects the capacity diagram. If not, repeat Steps 3-7 with a new value of $D_{i}$; otherwise go to Step 8 .

8. The earthquake-induced deformation demand is given by the $D$-value at the intersection point.

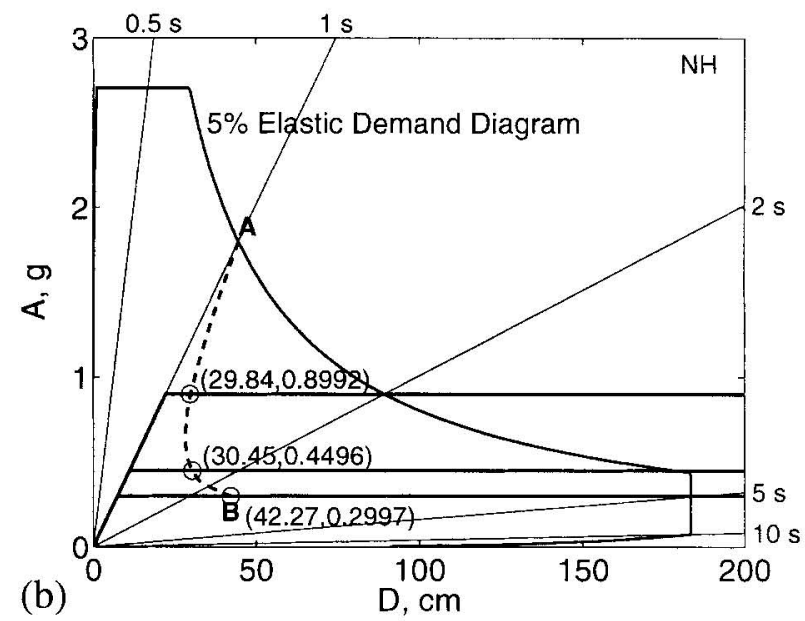

FIG. 7. Application of ATC-40 Procedure B for Elastic Design Spectrum: (a) Systems 1-3; (b) Systems 4-6
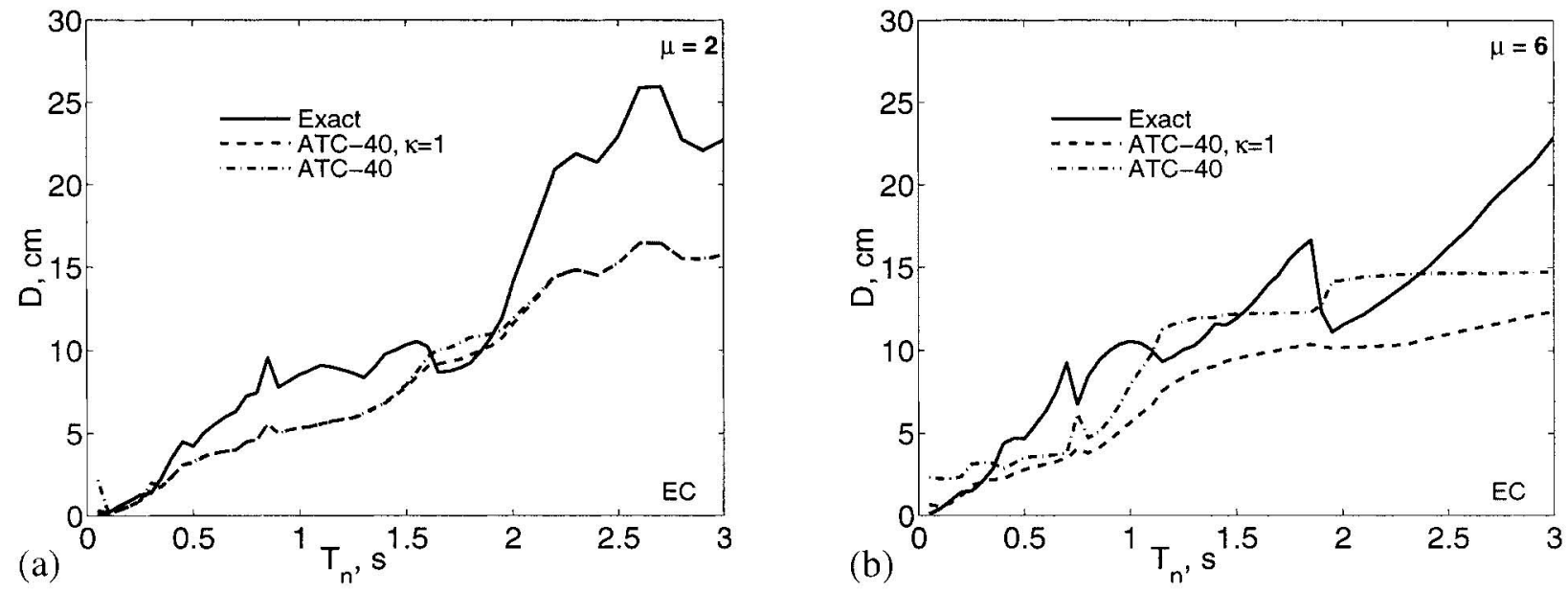

FIG. 8. Comparison of Deformations due to El Centro (EC) (1940) Ground Motion from Approximate Procedure and Nonlinear Response History Analysis: (a) $\mu=2$; (b) $\mu=6$
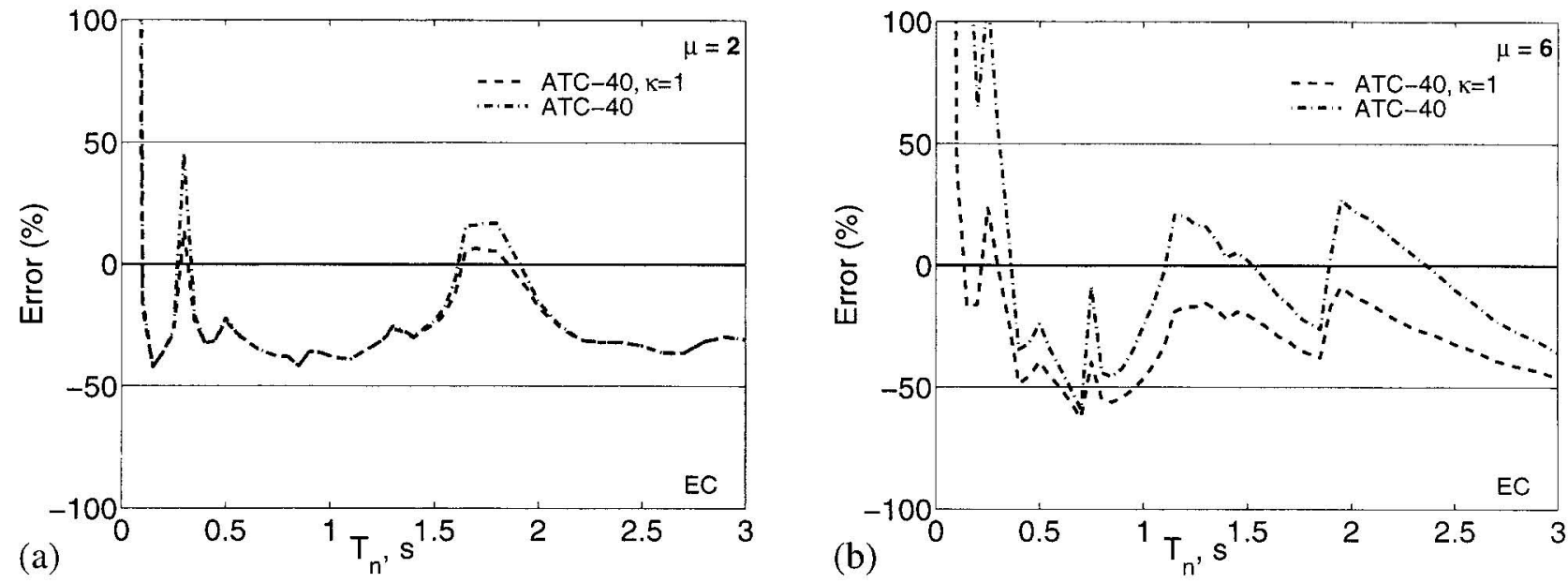

FIG. 9. Error in Deformations due to El Centro (EC) (1940) Ground Motion Computed by Approximate Procedure: (a) $\mu=2$; (b) $\mu=6$ 


\section{Examples}

Procedure B is implemented for Systems 1-6 (Table 1). For a number of assumed values of $\mu$ (or $D)$, pairs of values $D\left(T_{e q}\right.$, $\left.\hat{\zeta}_{e q}\right)$ and $A\left(T_{e q}, \hat{\zeta}_{e q}\right)$ are generated. These pairs are plotted to obtain the curve $A-B$ in Fig. 7, wherein capacity diagrams for three systems are shown together with the 5\%-damped linear elastic demand diagram; the latter need not be plotted. The results from this procedure are summarized in Table 1 and illustrated in Fig. 7 where the estimated deformations are noted. These approximate values are compared in Table 1 against the values determined directly from the inelastic design spectrum constructed by the procedure of Newmark and Hall (1982) as described in Chopra (1995, Section 7.10). Relative to these reference values, the discrepancy ranges from -5.2 to $-58.6 \%$ for the systems considered. Observe that the curve
$A-B$ provides the information to determine the deformation demand in several systems with the same $T_{n}$ values but different yield strengths. Detailed calculations for all results in Table 1 are available in Chopra and Goel (1999).

Procedure B always gives a unique estimate of the deformation, whereas the iterative Procedure A may not always converge as noted earlier. If it does converge, the two procedures gave the same value of deformation (within round-off and interpolation errors) for the bilinear systems analyzed.

\section{EVALUATION OF ATC-40 PROCEDURES}

\section{Specified Ground Motion}

The ATC-40 Procedure B is implemented for a wide range of system parameters and excitations in two versions: (1) $\kappa=$ 1 , i.e., the equivalent viscous damping is given by (4) and (5)

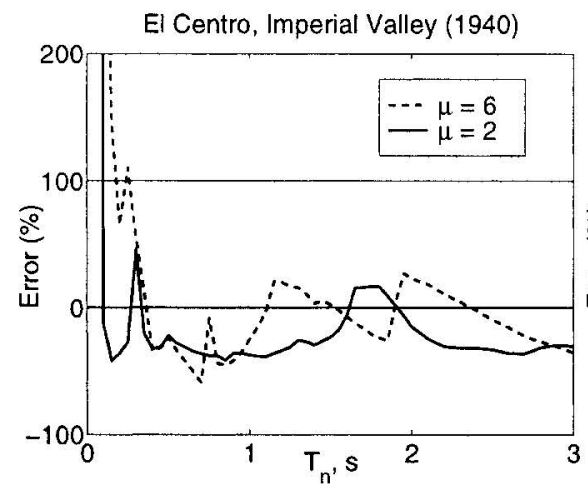

(a)

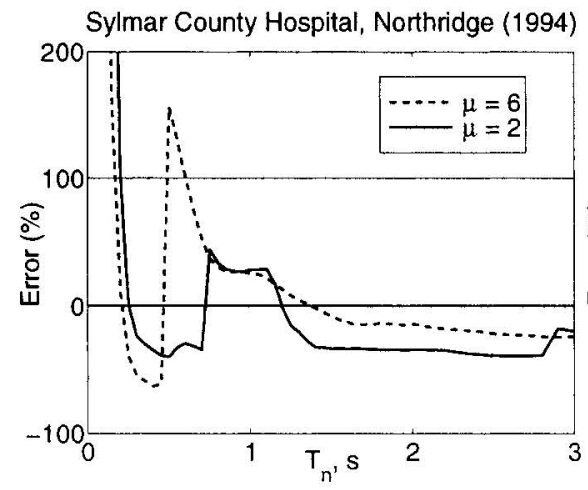

(c)

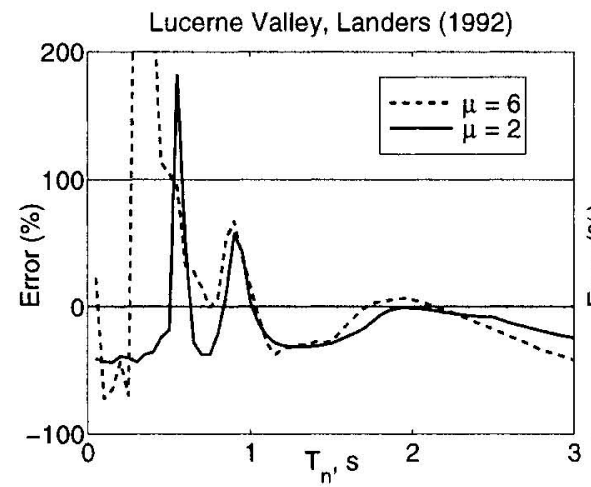

(e)

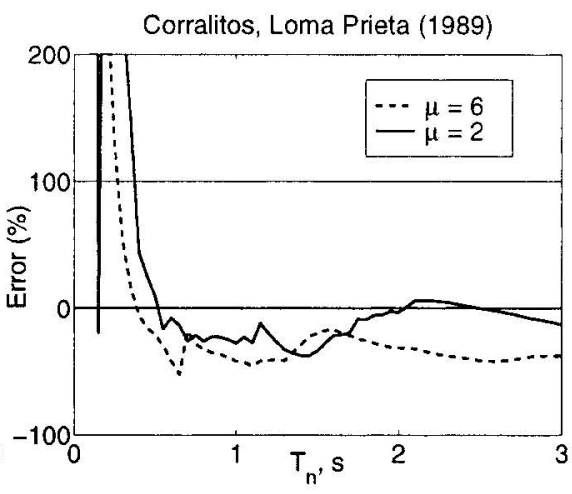

(b)

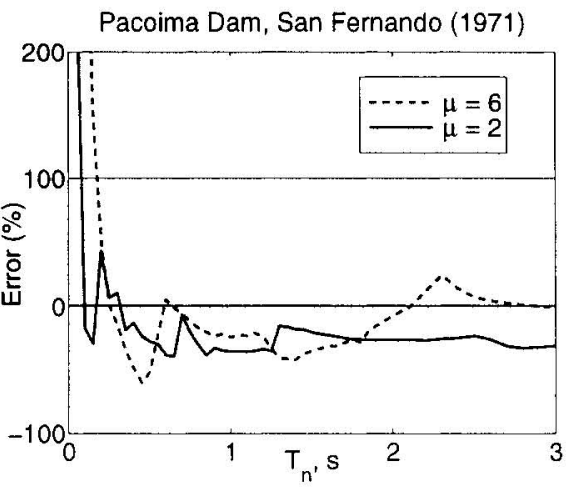

(d)

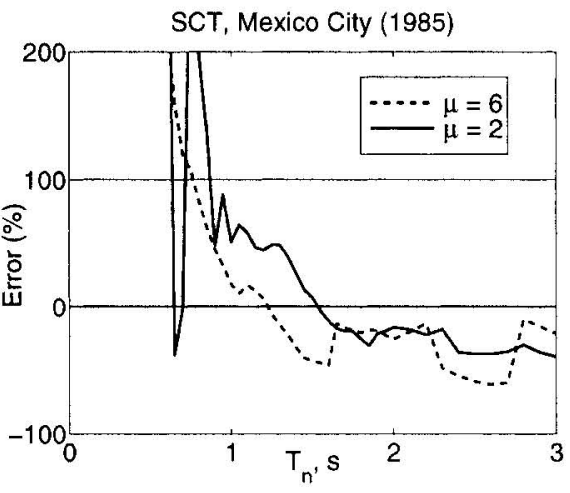

(f)

FIG. 10. Error in Deformations Computed by Approximate Procedure for Six Ground Motions 
based on well-established principles; and (2) $\kappa$ is given by Fig. 3 , a definition based primarily on judgment to account for different types of hysteretic behavior.

The yield strength of each elastoplastic system analyzed was chosen corresponding to an allowable ductility $\mu$

$$
f_{y}=\left(A_{y} / g\right) w
$$

where $w=$ weight of the system; and $A_{y}=$ pseudoacceleration corresponding to the allowable ductility and the vibration properties-natural period $T_{n}$ and damping ratio $\zeta$-of the system in its linear range of vibration. Recall that the ductility demand [computed by nonlinear response history analysis (RHA)] imposed by the selected ground motion on systems defined in this manner will exactly equal the allowable ductility (Chopra 1995, Section 19.1.1).

The peak deformation due to a selected ground motion determined by the ATC-40 method $D_{\text {approx }}$ is compared in Fig. 8 against the exact value $D_{\text {exact }}$ determined by RHA of the inelastic system, and the percentage error in the approximate result is plotted in Fig. 9. These figures permit several observations. The approximate procedure is not especially accurate. It underestimates significantly the deformation for wide ranges of $T_{n}$ values with errors approaching $50 \%$, implying that the estimated deformation is only about half of the value determined by nonlinear RHA. While inclusion of the damping modification factor $\kappa$ increases the estimated displacement, the accuracy of the approximate results improves only marginally for the smaller value of $\mu$. Therefore, the $\kappa$ factor is not attractive, particularly because it is based primarily on judgment.

Shown in Fig. 10 are the errors in the ATC-40 method, with the $\kappa$ factor included, for six different ground motions: (1) El Centro, S00E, 1940 Imperial Valley; (2) Corralitos, Chan-1, 90, 1989 Loma Prieta; (3) Sylmar County Hospital parking lot, Chan-3, 360', 1994 Northridge; (4) Pacoima Dam, N76W, 1971 San Fernando; (5) Lucerne Valley, S80W, 1992 Landers; and (6) SCT, S00E, 1985 Mexico City. Observe that, contrary to intuition, the error does not decrease consistently for smaller ductility. Whereas the magnitude of the error and its variation with $T_{n}$ depends on the excitation, the earlier observation that the error in the approximate method is significant is supported by results for several ground motions.

\section{Design Spectrum}

The ATC-40 Procedure B is implemented for a wide range of $T_{n}$ and $\mu$ values with the excitation characterized by the

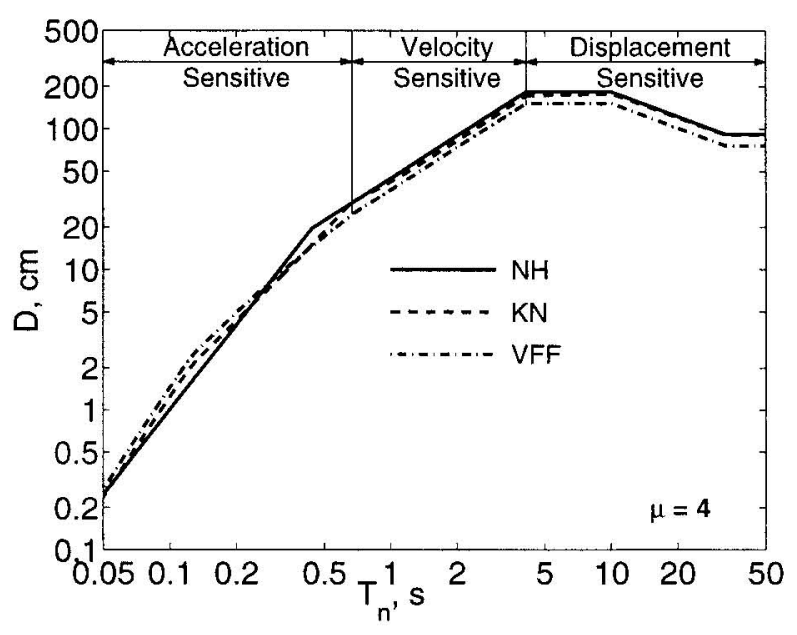

FIG. 11. Deformation of Inelastic Systems $(\mu=4)$ Determined from Inelastic Design Spectra using Three $R_{y}-\mu-T_{n}$ Equations: Newmark-Hall (NH), Krawinkler-Nassar (KN), and VidicFajfar-Fischinger (VFF) elastic design spectrum of Fig. 4. The yield strength was defined by (8) with $A_{y}$ determined from the inelastic design spectrum corresponding to the selected ductility factor. The resulting approximate values of deformations will be compared in this section with those determined directly from the design spectrum, as described next.

Given the properties $T_{n}, \zeta, f_{y}$, and $\alpha$ of the bilinear hysteretic system and the elastic design spectrum, the earthquake-in-

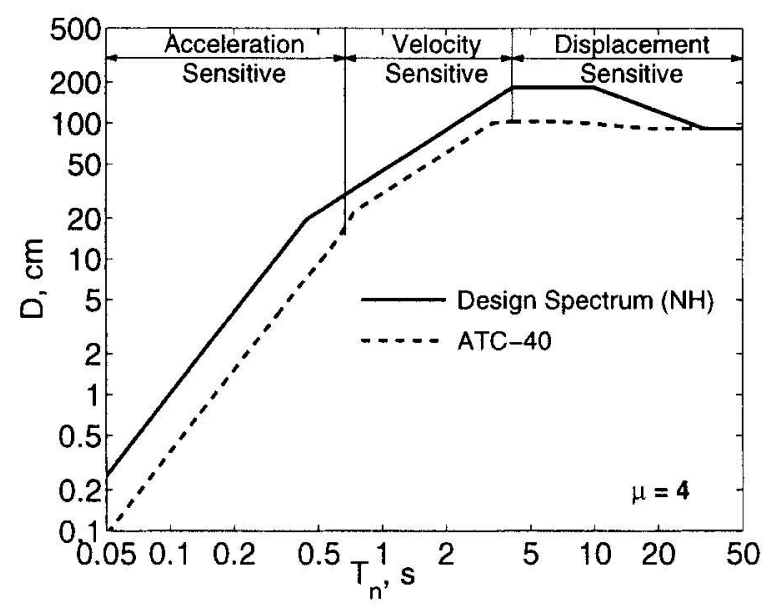

(a)

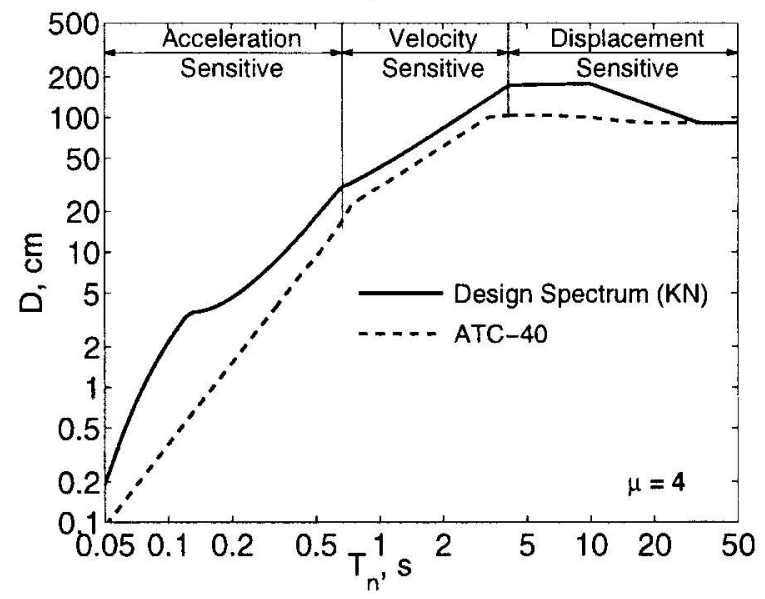

(b)

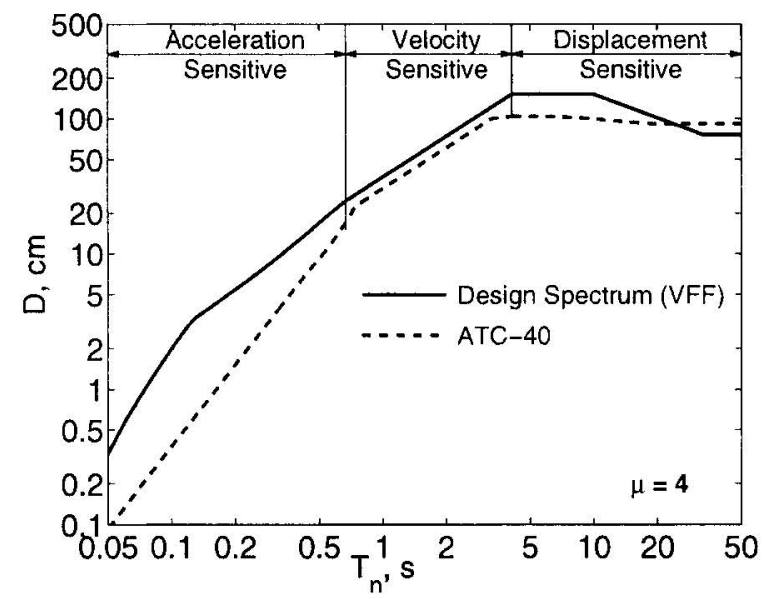

(c)

FIG. 12. Comparison of Deformations Computed by ATC-40 Procedure with Those from Three Different Inelastic Design Spectra $(\mu=4)$; (a) Newmark and Hall (NH) (1982); (b) Krawinkler and Nassar (KN) (1992); (c) Vidic et al. (VFF) (1994) 


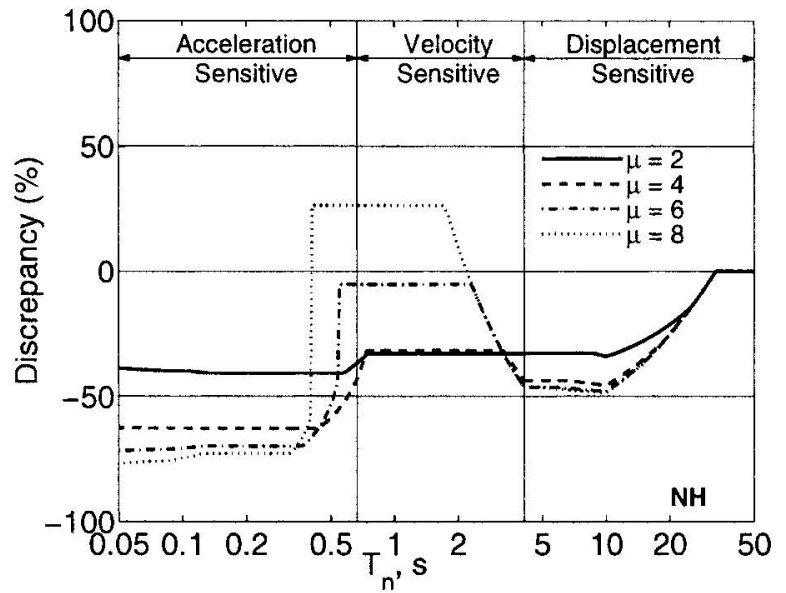

(a)

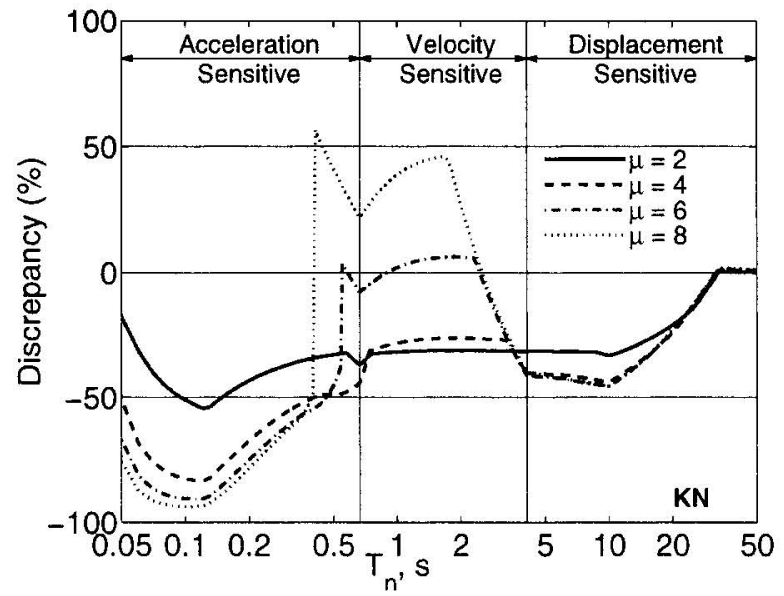

(b)

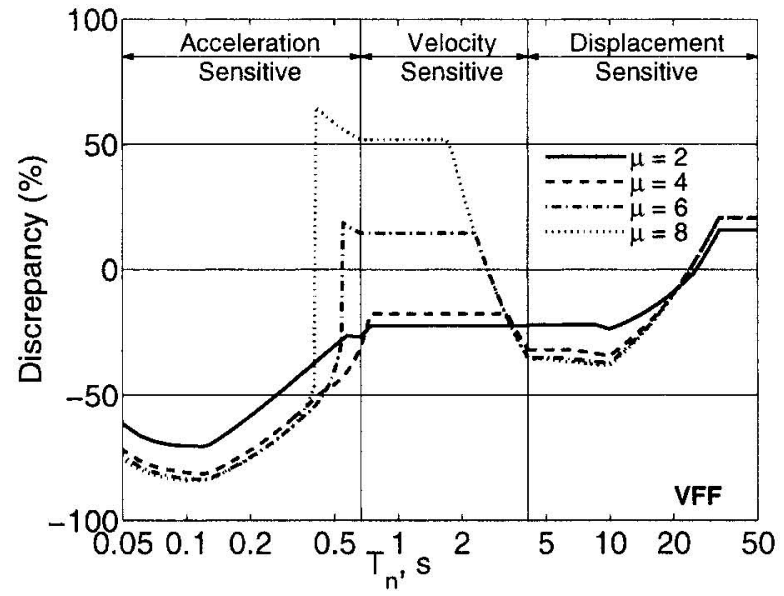

(c)

FIG. 13. Discrepancy in Deformations Computed by ATC-40 Procedure Relative to Three Different Inelastic Design Spectra: (a) Newmark and Hall (NH) (1982); (b) Krawinkler and Nassar (KN) (1992); (c) Vidic et al. (VFF) (1994)

duced deformation of the system can be determined directly from the design spectrum. The peak deformation $D$ of this system is given by

$$
D=\mu u_{y}
$$

with the yield deformation defined by

$$
u_{y}=\left(\begin{array}{c}
T_{n} \\
2 \pi
\end{array}\right)^{2} A_{y}
$$

where $A_{y}$ is related to the yield strength $f_{y}$ by (8). The yield strength reduction factor is given by

$$
R_{y}=\begin{aligned}
& f_{o} \\
& f_{y}
\end{aligned}=\begin{aligned}
& A \\
& A_{y}
\end{aligned}
$$

where

$$
f_{o}=\left(\begin{array}{l}
A \\
g
\end{array}\right) w
$$

is the minimum yield strength required for the structure to remain elastic; and $A=$ pseudoacceleration ordinate of the elastic design spectrum at $\left(T_{n}, \zeta\right)$. Putting (9), (10), and (12) together gives

$$
D=\mu \frac{1}{R_{y}}\left(\begin{array}{c}
T_{n} \\
2 \pi
\end{array}\right)^{2} A
$$

Eq. (13) provides a convenient way to determine the deformation of the inelastic system from the design spectrum. All that remains to be done is to determine $\mu$ for a given $R_{y}$; the latter is known from (11) for a structure with known $f_{y}$.

Presented in Fig. 11 are the deformations determined by (13) using three different $R_{y}-\mu-T_{n}$ equations: Newmark and Hall (1982), Krawinkler and Nassar (1992) for elastoplastic systems, and Vidic et al. (1994) for bilinear systems. The equations describing these relationships are summarized in Chopra and Goel (1999). Observe that the three recommendations lead to similar results except for $T_{n}<0.3 \mathrm{~s}$, indicating that the inelastic design spectrum is a reliable approach to estimate the earthquake-induced deformation of yielding systems.

The deformation estimates by the ATC-40 method are compared in Fig. 12 with those from inelastic design spectra presented in Fig. 11. Relative to these reference values, the percentage discrepancy in the approximate result is plotted in Fig. 13. The results of Figs. 12 and 13 permit the following observations. The approximate procedure leads to significant discrepancy, except for very long periods $\left(T_{n}>T_{f}\right.$ in Fig. 4$)$. The magnitude of this discrepancy depends on the design ductility and the period region. In the acceleration-sensitive $\left(T_{n}<T_{c}\right)$ and displacement-sensitive $\left(T_{d}<T_{n}<T_{f}\right)$ regions (Fig. 4), the approximate procedure significantly underestimates the deformation; the discrepancy increases with increasing $\mu$. In the velocity-sensitive $\left(T_{c}<T_{n}<T_{d}\right)$ region, the ATC-40 procedure significantly underestimates the deformation for $\mu=2$ and 4 , but overestimates it for $\mu=8$ and is coincidentally accurate for $\mu=6$.

In passing, note that the ATC-40 procedure is deficient relative to even the elastic design spectrum in the velocity-sensitive and displacement-sensitive regions $\left(T_{n}>T_{c}\right)$. For $T_{n}$ in these regions, the peak deformation of an inelastic system is the same as that of the corresponding linear system, which is the well-known equal displacement rule (Veletsos and Newmark 1960; Chopra 1995, p. 272). The peak deformation of a linear system can therefore be estimated from the elastic design spectrum. However, the ATC-40 procedure requires analyses of several equivalent linear systems and still produces worse results.

\section{CONCLUSIONS}

This investigation of capacity-demand-diagram methods to estimate the earthquake-induced deformation of inelastic SDF systems has led to the following conclusions:

- Based on the belief that the deformation of an inelastic system can be estimated by an iterative method requiring analysis of a sequence of equivalent linear systems, the 
ATC-40 Procedure A did not converge for some of the systems analyzed. It converged in many cases but not to the exact deformation determined by nonlinear RHA of the inelastic system, nor to the value determined from the inelastic design spectrum. Thus, convergence of this iterative procedure is deceptive because it can leave erroneous impression that the calculated deformation is accurate. This approximate procedure therefore does not meet the basic requirement of a rational iterative procedure: it should always converge to the exact result after sufficient number of iterations.

- The ATC-40 Procedure B always gives a unique value of deformation, same as determined by Procedure A if it converged.

- The peak deformation of inelastic systems, determined by ATC-40 procedures, when compared against results of nonlinear RHA for several ground motions, were shown to be inaccurate. The approximate procedure underestimates significantly the deformation for a wide range of $T_{n}$ values with errors approaching $50 \%$, implying that the estimated deformation is only about half of the exact value.

- The damping modification factor $\kappa$ in ATC-40 procedures improves the deformation estimate only marginally. Therefore, the $\kappa$ factor is not attractive, particularly because it is based primarily on judgment.

- The ATC-40 procedures were implemented for a wide range of $T_{n}$ and $\mu$ values with the excitation characterized by an elastic design spectrum. The resulting estimate of deformation for the inelastic system was compared with the deformation determined from the inelastic design spectrum using three different $R_{y}-\mu-T_{n}$ equations (Newmark and Hall 1982; Krawinkler and Nassar 1992; Vidic et al. 1994), all of which provided similar results. Relative to these references values, the approximate procedure significantly underestimates the deformation for a wide range of $T_{n}$ and $\mu$ values.

- The ATC-40 procedures are deficient relative to even the elastic design spectrum in estimating the peak deformation of an inelastic system with $T_{n}$ in the velocity-sensitive or displacement-sensitive regions of the spectrum.

\section{ACKNOWLEDGMENTS}

This research investigation was funded by the National Science Foundation under Grant CMS-9812531, a part of the U.S.-Japan Cooperative Research in Urban Earthquake Disaster Mitigation. This financial support is gratefully acknowledged. The writers have benefited from discussions with Chris D. Poland and Wayne A. Low, who are working on a com- panion research project awarded to Degenkolb Engineers; and with Sigmund A. Freeman, who, more than any other individual, has been responsible for the concept and development of the capacity spectrum method.

\section{APPENDIX. REFERENCES}

ATC. (1996). "Seismic evaluation and retrofit of concrete buildings." Rep. ATC-40, Applied Technology Council, Redwood City, Calif.

Chopra, A. K. (1995). Dynamics of structures: Theory and applications to earthquake engineering. Prentice-Hall, N.J.

Chopra, A. K., and Goel, R. K. (1999). "Capacity-demand-diagram methods for estimating seismic deformation of inelastic structures: SDF systems." Rep. No. PEER-1999/02, Pacific Earthquake Engrg. Res. Ctr., University of California, Berkeley, Calif.

Deierlein, G. G., and Hsieh, S.-H. (1990). "Seismic response of steel frames with semi-rigid connections using the capacity spectrum method." Proc., 4th U.S. Nat. Conf. on Earthquake Engrg., Vol. 2, $863-872$.

FEMA. (1997). "NEHRP commentary on the guidelines for the seismic rehabilitation of buildings." FEMA-274, Federal Emergency Management Agency, Washington, D.C.

Freeman, S. A. (1978). "Prediction of response of concrete buildings to severe earthquake motion." Publ. SP-55, American Concrete Institute, Detroit, 589-605.

Freeman, S. A., Nicoletti, J. P., and Tyrell, J. V. (1975). "Evaluations of existing buildings for seismic risk $A$ case study of Puget Sound Naval Shipyard, Bremerton, Washington." Proc., 1st U.S. Nat. Conf. on Earthquake Engrg., 113-122.

Hudson, D. E. (1965). "Equivalent viscous friction for hysteretic systems with earthquake-like excitations." Proc., 3rd World Conf. on Earthquake Engrg., Vol. II, 185-202.

Iwan, W. D., and Gates, N. C. (1979). "Estimating earthquake response of simple hysteretic structures." J. Engrg. Mech. Div., ASCE, 105(3), 391-405.

Jennings, P. C. (1968). "Equivalent viscous damping for yielding structures." J. Engrg. Mech. Div., ASCE, 94(1), 103-116.

Krawinkler, H. K., and Nassar, A. A. (1992). "Seismic design based on ductility and cumulative damage demands and capacities." Nonlinear seismic analysis and design of reinforced concrete buildings, P. Fajfar and H. Krawinkler, eds., Elsevier Science, New York.

Newmark, N. M., and Hall, W. J. (1982). Earthquake spectra and design. Earthquake Engineering Research Institute, Berkeley, Calif.

Paret, T. F., Sasaki, K. K., Eilbekc, D. H., and Freeman, S. A. (1996). "Approximate inelastic procedures to identify failure mechanisms from higher mode effects." 11th World Conf. on Earthquake Engrg.

Reinhorn, A. M., Li, C., and Constantinou, M. C. (1995). "Experimental and analytical investigations of seismic retrofit of structures with supplemental damping." Rep. No. NCEER-95-0001, State University of New York at Buffalo, Buffalo, N.Y.

Veletsos, A. S., and Newmark, N. M. (1960). "Effects of inelastic behavior on the response of simple system to earthquake motions." Proc., 2nd World Conf. on Earthquake Engrg., Vol. 2, 895-912.

Vidic, T., Fajfar, P., and Fischinger, M. (1994). "Consistent inelastic design spectra: Strength and displacement." Earthquake Engrg. and Struct. Dyn., 23(5), 507-521. 